\author{
Sigrid Leyendecker ${ }^{2}$ \\ Biocomputing Group, \\ Berlin Mathematical School, \\ Free University of Berlin, \\ Berlin 14195, Germany \\ e-mail: sleye@zedat.fu-berlin.de \\ Leonard J. Lucas \\ Graduate Aeronautical Laboratories, \\ California Institute of Technology, \\ Pasadena, CA 91125 \\ e-mail: lenny.lucas@gmail.com \\ Houman Owhadi \\ Department of Applied and Computational \\ Mathematics and Department of Control and \\ Dynamical Systems, \\ California Institute of Technology, \\ Pasadena, CA 91125 \\ e-mail: owhadi@caltech.edu \\ Michael Ortiz \\ Graduate Aeronautical Laboratories, \\ California Institute of Technology, \\ Pasadena, CA 91125 \\ e-mail: ortiz@aero.caltech.edu
}

\section{Optimal Control Strategies for Robust Certification ${ }^{1}$}

We present an optimal control methodology, which we refer to as concentration-ofmeasure optimal control (COMOC), that seeks to minimize a concentration-of-measure upper bound on the probability of failure of a system. The systems under consideration are characterized by a single performance measure that depends on random inputs through a known response function. For these systems, concentration-of-measure upper bound on the probability of failure of a system can be formulated in terms of the mean performance measure and a system diameter that measures the uncertainty in the operation of the system. COMOC then seeks to determine the optimal controls that maximize the confidence in the safe operation of the system, defined as the ratio of the design margin, which is measured by the difference between the mean performance and the design threshold, to the system uncertainty, which is measured by the system diameter. This strategy has been assessed in the case of a robot-arm maneuver for which the performance measure of interest is assumed to be the placement accuracy of the arm tip. The ability of COMOC to significantly increase the design confidence in that particular example of application is demonstrated. [DOI: 10.1115/1.4001375]

\section{Introduction}

The objective of this work is the development of an optimal control methodology for the minimization of the probability of failure of a system. Thus, we consider systems that are stochastic and whose operation can succeed or fail with a certain probability. In addition, the operation of the system depends on a certain set of control variables. For these systems, the mathematical and computational problems that we address concerns the determination of optimal control laws that result in the least possible probability of failure of the system.

Often, the probability of failure of a system-and its dependence on the control variables-is not known. However, for certain classes of systems, upper bounds of the probability of failure can be formulated - and computed—with some generality. For instance, consider systems that are deterministic except for the randomness of their inputs $X$. Suppose, in addition, that the safe operation of the system requires that a certain performance measure $Y$ be below a threshold $a$, and that the performance measure depends on the inputs through a response function $F(X)$. Under these assumptions, concentration-of-measure inequalities (cf. e.g., [1-3], Sec. 2 of this paper for a brief review) provide convenient upper bounds for the probability of failure of the system. These upper bounds are attractive because they depend solely on two quantities: the mean performance of the system and a system diameter that measures the uncertainty in the operation of the system. The computations of both parameters is straightforward, albeit possibly costly: the mean performance can be computed by Monte Carlo sampling and the diameter by a global optimization

\footnotetext{
${ }^{1}$ Submitted to the ASME Journal of Computational and Nonlinear Dynamics, Submitted to the ASME Journal of Computational and Nonlinear Dynamics,
special issue on Multidisciplinary High-Performance Computational Multibody Dynamics, edited by Dan Negrut and Olivier Bauchau, Mar. 15, 2009.

${ }^{2}$ Corresponding author.

Contributed by the Design Engineering Division of ASME for publication in the Journal of Computational and Nonlinear Dynamics. Manuscript received March 16, 2009; final manuscript received October 26, 2009; published online May 18, 2010. Assoc. Editor: Dan Negrut.
}

over the space of inputs. In lieu of an exact probability of failure, we may instead seek optimal controls that minimize a probability of failure upper bound, such as supplied by concentration-ofmeasure inequalities. The proposed methodology, called concentration-of-measure optimal control (COMOC), is introduced in Sec. 3. The resulting optimal controls then maximize the design margin, i.e., the difference between the threshold and the mean performance for safe operation, or reduce the uncertainty in the operation of the system, as measured by the system diameter, or both.

We assess the COMOC in a specific area of application: positioning accuracy in robotic-arm maneuvers, modeled as threedimensional systems of rigid bodies [4,5]. The system is made stochastic by first assuming that the lengths of various segments of the arm are random, and second, that in addition, the system experiences random forcing due to side wind. We investigate a particular robot-arm maneuver whose successful operation requires a minimum arm tip positioning accuracy, both by deterministic analysis of the nominal geometry of the system without wind forces and by COMOC. For completeness, a brief account of the discrete mechanics and optimal control for constrained systems (DMOCC) methodology employed in the deterministic calculations is included in Sec. 4. DMOCC is a direct transcription method transforming the optimal control problem into a constrained optimization problem, where the boundary conditions and the discrete equations of motion serve as equality constraints. In particular, DMOCC is designed for mechanical systems whose dynamics itself is holonomically constrained. The results of numerical experiments are collected in Sec. 5. In the particular example under consideration, COMOC reduces the concentrationof-measure probability of failure upper bound by about one order of magnitude with respect to the deterministic optimal control.

\section{Concentration-of-Measure Inequalities for Uncer- tainty Quantification and Certification}

The application of concentration-of-measure inequalities for uncertainty quantification and certification of engineering systems 
is relatively new [3]. For completeness, we proceed to give a brief account of concentration-of-measure inequalities as they bear on the type of systems and applications under consideration here.

The goal is to certify whether a system is likely to perform safely and reliably within the design specifications. Suppose that the system operates safely if its performance measure $Y \in A \subset E$ is in the admissible set $A$ and is considered to fail if $Y \in A^{c}=E \backslash A$ is in the inadmissible set $A^{c}$, where $E$ is an Euclidian space. For systems characterized by a single performance measure, the admissible set often is of the form $A=(-\infty, a]$, where $a$ is the threshold for the safe operation of the system. The system is certified when the probability of failure $\mathbb{P}\left[Y \in A^{c}\right]$ is less than a prespecified tolerance $\epsilon$, i.e., if

$$
\mathbb{P}\left[Y \in A^{c}\right]<\epsilon
$$

Often, however, the probability of failure of a system is not known, and its direct computation, e.g., by Monte Carlo sampling, is prohibitively expensive. Such is the case, for instance, of systems of large dimensionality for which failure is a rare event. In these cases, rigorous certification can still be achieved if a probability of failure upper bound can be determined, namely, by requiring that the probability of failure upper bound be less than the tolerance $\epsilon$. For systems whose randomness can be characterized by means of random inputs, a convenient class of upper bounds is supplied by concentration-of-measure inequalities, which we briefly summarize. A rigorous certification methodology can then be formulated based on such concentration-of-measure inequalities [3].

For present purposes, it suffices to assume that a perfect model for the system's response is available, i.e., a mathematical model that describes the system exactly. In particular, errors stemming from numerical approximations are neglected. For simplicity, we restrict attention to the quantification of uncertainty of a single performance measure $Y \in E$ and assume that the relation $Y$ $=F(X)$ describes the system exactly in terms of the random vector $X: \Omega \rightarrow \chi_{1} \times \ldots \times \chi_{M}$, where $(\Omega, \mathcal{U}, \mathbb{P})$ is a probability space [6]. Let $\mathbb{E}[Y]$ denote the mean performance of the system and assume that it belongs to the interior of $A$. Then, if $F$ is integrable and the input parameters are independent, McDiarmid's inequality [7] states that

$$
\mathrm{P}[Y-\mathbb{E}[Y] \leq-r] \leq \exp \left(-2 \frac{r^{2}}{D_{F}^{2}}\right)
$$

where $r \geq 0$ and the diameter of the system is defined as

$$
\begin{aligned}
D_{F}^{2}= & \sum_{k=1}^{M} \sup _{X_{1}, \ldots, X_{k-1}, X_{k+1}, \ldots, X_{M} \in \mathcal{X}_{1} \times \ldots \mathcal{X}_{k-1} \times \mathcal{X}_{k+1}, \ldots, \times \mathcal{X}_{M}} \\
& \times \sup _{\left(A_{k}, B_{k}\right) \in \mathcal{X}_{k}^{2}}\left|F\left(X_{1}, \ldots, A_{k}, \ldots, X_{M}\right)-F\left(X_{1}, \ldots, B_{k}, \ldots, X_{M}\right)\right|^{2}
\end{aligned}
$$

Thus, the diameter is the sum of the maximum squared oscillation in response from a random variable pair (independent and identically distributed) varying in turn when all random variables are allowed to vary over their entire ranges, and provides a measure of the uncertainty in the operation of the system. Using $r=(a$ $-\mathbb{E}[Y])_{+}=\max (0, a-\mathbb{E}[Y])$, the bound (2) can be rewritten as an upper bound on the probability of failure

$$
\mathbb{P}\left[Y \in A^{c}\right] \leq \exp \left(-2 \frac{(a-\mathbb{E}[Y])_{+}^{2}}{D_{F}^{2}}\right)
$$

Often, however, the mean performance $\mathbb{E}[Y]$ is not known a priori and must be estimated. For instance, the mean performance can be estimated by performing $m$ evaluations of the model $F(X)$ based on unbiased Monte Carlo sampling of the input parameters, resulting in predicted performance measures $Y^{1}, \ldots, Y^{m}$. The corresponding mean performance estimate is

$$
\langle Y\rangle=\frac{1}{m} \sum_{i=1}^{m} Y^{i}
$$

When the mean performance is estimated by sampling, the probability of failure can only be determined to within a predefined estimation tolerance $\epsilon^{\prime}$ reflecting the randomness of $\langle Y\rangle$. Specifically, if

$$
\alpha=D_{F} m^{-1 / 2}\left(-\log \epsilon^{\prime}\right)^{1 / 2}
$$

then, with probability $1-\epsilon^{\prime}$

$$
\mathrm{P}\left[Y \in A^{c}\right] \leq \exp \left(-2 \frac{(a-\langle Y\rangle-\alpha)_{+}^{2}}{D_{F}^{2}}\right)
$$

A rigorous certification criterion can now be obtained by requiring that this bound be less than the probability of failure tolerance, with the result

$$
\mathrm{CF} \equiv \frac{M}{U} \equiv \frac{(a-\langle Y\rangle-\alpha)_{+}}{D_{F}} \geq \sqrt{\log \sqrt{\frac{1}{\epsilon}}}
$$

Here $M=(a-\langle Y\rangle-\alpha)_{+}$may be interpreted as a design margin, $U$ $=D_{F}$ as a measure of the uncertainty in the operation of the system, and $\mathrm{CF}$ as a confidence factor. Certification then requires the confidence factor $(\mathrm{CF})$ to be in excess of the value $\sqrt{\log \sqrt{(1 / \epsilon)}}$. It is interesting to observe, comparing Eq. (6) to Eq. (4), that the estimation of the mean performance reduces the margin by the value $\alpha$ to account for statistical deviations. This margin hit can be reduced to an arbitrary small value by carrying out a sufficiently large number of model evaluations.

It is instructive to compare the probabilities of failure bounds obtained from concentration-of-measure inequalities with those determined directly by random sampling. Consider an empirical probability measure

$$
\mu_{m}=\frac{1}{m} \sum_{i=1}^{m} \delta_{Y^{i}}
$$

obtained via traditional random sampling methods. Here

$$
\delta_{Y}= \begin{cases}1 & \text { for } Y \in A^{c} \\ 0 & \text { for } Y \in A\end{cases}
$$

Then Hoeffding's inequality [8] gives

$$
\mathrm{P}\left[Y \in A^{c}\right] \leq \mu_{m}\left[A^{c}\right]+\sqrt{\frac{1}{2 m} \log \frac{1}{\epsilon^{\prime}}}
$$

with probability $1-\epsilon^{\prime}$. This bound reveals that the number of experiments required to certify a system based on statistical sampling alone is of the order of $(1 / 2) \epsilon^{-2} \log \left(1 / \epsilon^{\prime}\right)$. For computationally expensive models, the number of function evaluations becomes restrictive and unreasonable as $\epsilon^{\prime}$ decreases. By contrast, the diameter in the concentration-of-measure inequality (6) is independent of $\epsilon$, which confers concentration-of-measure inequalities a considerable advantage when failure is a rare event and the required probability of failure is low.

\section{Concentration-of-Measure Optimal Control}

With this concept, to compute for probability of failure upper bounds at hand, one can design the system such that confidence in its safe operation is improved via COMOC.

Suppose that the system under consideration is a controlled dynamical system with time-dependent states $\boldsymbol{x}:\left[t_{0}, t_{N}\right] \rightarrow \mathbb{R}^{n_{x}}$ and controls $\boldsymbol{\tau}:\left[t_{0}, t_{N}\right] \rightarrow \mathbb{R}^{n_{\tau}}$, where $t \in\left[t_{0}, t_{N}\right] \subset \mathbb{R}$ denotes the time and $N, n_{x}, n_{\tau}, n_{h} \in \mathbb{N}$. Let the dynamical system be specified by

$$
\begin{gathered}
\dot{\boldsymbol{x}}(t)=\boldsymbol{\phi}(\boldsymbol{x}(t), \boldsymbol{\tau}(t)) \\
\boldsymbol{x}\left(t_{0}\right)=\boldsymbol{x}_{0} \\
\boldsymbol{h}(\boldsymbol{x}(t))=\mathbf{0}
\end{gathered}
$$


with the smooth function $\boldsymbol{\phi}: \mathbb{R}^{n_{x}} \times \mathbb{R}^{n_{\tau}} \rightarrow \mathbb{R}^{n_{x}}$, the initial value $\boldsymbol{x}_{0}$ $\in \mathbb{R}^{n_{x}}$, and the path constraints $\boldsymbol{h}: \mathbb{R}^{n_{x}} \rightarrow \mathbb{R}^{n_{h}}$. In general, the quantification of uncertainty of a performance measure $Y$ and the corresponding $\mathrm{CF}$ are dependent on the complete system, i.e., on $\boldsymbol{x}_{0}, \boldsymbol{\phi}, \boldsymbol{h}$ and $\boldsymbol{\tau}$, and one could aim at improving the system such that the probability of failure decreases. However, in many practical situations, one has to deal with the given system equations, initial conditions, and path constraints, but can manipulate the controls more easily. Therefore, we seek to determine the optimal control laws that result in the least possible probability of failure by identifying the objective function for optimal control with the probability of failure $\mathrm{P}[Y>a]$. Often, however, the probability $\mathbb{P}[Y>a]$ is not known explicitly. In these cases, we seek instead to minimize a concentration-of-measure upper bound of the probability of failure such as Eq. (6).

Indicate the dependence of the performance measure on a given control law by $Y^{\tau}=F^{\tau}(X)$. Then one is faced with the following optimal control problem:

$$
\min _{\boldsymbol{\tau}(\cdot)}(-\mathrm{CF}(\boldsymbol{\tau})) \equiv-\frac{M^{\tau}}{U^{\tau}} \equiv-\frac{\left(a-\left\langle Y^{\tau}\right\rangle-\alpha^{\tau}\right)_{+}}{D_{F^{\tau}}}
$$

subject to Eq. (8), where the system equations, initial conditions, and path constraints serve as constraints for the optimization. Evidently, by this choice of objective function the optimal control $\tau$ seeks to maximize confidence in the safe operation of the systems either by increasing the placement margin $M^{\tau}$, i.e., by decreasing $a-\left\langle Y^{\tau}\right\rangle$, or by reducing the uncertainty $U^{\tau}$ of the maneuver, i.e., by reducing the diameter $D_{F}$, or both.

\section{Discrete Mechanics and Optimal Control of Con- strained Multibody Dynamics}

The equations of motion of a controlled mechanical system subject to holonomic constraints may be formulated in terms of the states and controls by applying a constrained version of the Lagrange-d'Alembert principle. DMOCC, a structure preserving scheme for the optimal control of such systems, is derived in from Refs. [4,5], using a discrete analog of that principle. Structure preservation is inherited when the system is reduced to its minimal dimension by the discrete null space method. Together with the initial and final conditions on the configuration and conjugate momentum, the reduced discrete equations serve as nonlinear equality constraints for the minimization of a given objective functional. The algorithm yields a sequence of discrete configurations together with a sequence of actuating forces, optimally guiding the system from the initial to the desired final state. In particular, for the optimal control of three-dimensional multibody systems, a force formulation consistent with the joint constraints is introduced in Ref. [5], and consistency of the evolution of momentum maps is proved for different types of joints. OberBlöbaum et al. [9] focused on the analysis of discrete mechanics and optimal control (DMOC in the unconstrained case) and gives a proof of convergence of the DMOC-solution to that of the original (continuous) optimal control problem.

In this section, the formulation of DMOCC is summarized briefly. Then, it is described how the robot arm is modeled as a spatially fixed spherical pair, and a short overview on the main ingredients for the optimal control of the robot arm is given. Finally, DMOCC will be used to determine a cost minimizing robotarm maneuver in the deterministic setting.

4.1 DMOCC. The equations of motion for forced, holonomically constrained systems can be derived via a variational principle. Quite different strategies for the treatment of the constraints are at the disposal. One possibility described for conservative systems in Ref. [10] is to transform the differential algebraic equations (DAEs) by a null space method with reparameterization. Analogous steps can be performed in the temporal discrete variational setting to derive the forced constrained discrete Euler-
Lagrange equations and their reduction to minimal dimensions. Again, these steps have been investigated in detail in Ref. [10] for conservative systems and in Ref. [5] for forced systems, and the method is summarized here.

Consider an $n$-dimensional mechanical system with the timedependent configuration vector $\boldsymbol{q}(t) \in Q$ and velocity vector $\dot{\boldsymbol{q}}(t)$ $\in T_{\boldsymbol{q}(t)} Q$ in the tangent space $T_{\boldsymbol{q}(t)} Q$ to the configuration manifold $Q$. Let the configuration be constrained by the function $\boldsymbol{g}(\boldsymbol{q})=\mathbf{0}$ $\in \mathbb{R}^{m}$ with constraint manifold $C=\{\boldsymbol{q} \in Q \mid \boldsymbol{g}(\boldsymbol{q})=\mathbf{0}\}$ and influenced by the force field $f: \mathbb{R}^{n-m} \times T Q \rightarrow T^{*} Q$.

Corresponding to the configuration manifold $Q$, the discrete phase space is defined by $Q \times Q$, which is locally isomorphic to $T Q$. For a constant time-step $h \in \mathbb{R}$, a path $\boldsymbol{q}:\left[t_{0}, t_{N}\right] \rightarrow Q$ is replaced by a discrete path $\boldsymbol{q}_{d}:\left\{t_{0}, t_{0}+h, \ldots, t_{0}+N h=t_{N}\right\} \rightarrow Q(N$ $\in \mathbb{N})$, where $\boldsymbol{q}_{n}=\boldsymbol{q}_{d}\left(t_{n}\right)$ is viewed as an approximation to $\boldsymbol{q}\left(t_{n}\right)$ at $t_{n}=t_{0}+n h$. The action integral is approximated in a time interval $\left[t_{n}, t_{n+1}\right]$ using the discrete Lagrangian $L_{d}: Q \times Q \rightarrow \mathbb{R}$ and the discrete constraint function $\boldsymbol{g}_{d}: Q \rightarrow \mathbb{R}$. Similarly, $\boldsymbol{\lambda}_{n}=\boldsymbol{\lambda}_{d}\left(t_{n}\right)$ approximates the Lagrange multiplier, while the force field $\boldsymbol{f}$ is approximated by two discrete forces $f_{n}^{-}, f_{n}^{+}: T^{*} U \times Q \rightarrow T^{*} Q$.

4.1.1 Discrete Constrained Lagrange-d'Alembert Principle. The discrete version of the constrained Lagrange-d'Alembert principle requires the discrete path $\left\{\boldsymbol{q}_{n}\right\}_{n=0}^{N}$ and multipliers $\left\{\boldsymbol{\lambda}_{n}\right\}_{n=0}^{N}$ to fulfill

$$
\begin{aligned}
& \delta \sum_{n=0}^{N-1}\left(L_{d}\left(\boldsymbol{q}_{n}, \boldsymbol{q}_{n+1}\right)-\frac{1}{2} \boldsymbol{g}_{d}^{T}\left(\boldsymbol{q}_{n}\right) \cdot \boldsymbol{\lambda}_{n}-\frac{1}{2} \boldsymbol{g}_{d}^{T}\left(\boldsymbol{q}_{n+1}\right) \cdot \boldsymbol{\lambda}_{n+1}\right)+\sum_{n=0}^{N-1}\left(\boldsymbol{f}_{n}^{-} \cdot \delta \boldsymbol{q}_{n}\right. \\
& \left.\quad+\boldsymbol{f}_{n}^{+} \cdot \delta \boldsymbol{q}_{n+1}\right)=0
\end{aligned}
$$

for all variations $\left\{\delta \boldsymbol{q}_{n}\right\}_{n=0}^{N}$ and $\left\{\delta \boldsymbol{\lambda}_{n}\right\}_{n=0}^{N}$ with $\delta \boldsymbol{q}_{0}=\delta \boldsymbol{q}_{N}=\mathbf{0}$, which is equivalent to the constrained forced discrete Euler-Lagrange equations

$$
\begin{gathered}
D_{2} L_{d}\left(\boldsymbol{q}_{n-1}, \boldsymbol{q}_{n}\right)+D_{1} L_{d}\left(\boldsymbol{q}_{n}, \boldsymbol{q}_{n+1}\right)-\boldsymbol{G}_{d}^{T}\left(\boldsymbol{q}_{n}\right) \cdot \boldsymbol{\lambda}_{n}+\boldsymbol{f}_{n-1}^{+}+\boldsymbol{f}_{n}^{-}=\mathbf{0} \\
\boldsymbol{g}\left(\boldsymbol{q}_{n+1}\right)=\mathbf{0}
\end{gathered}
$$

for $n=1, \ldots, N-1$, where $\boldsymbol{G}_{d}\left(\boldsymbol{q}_{n}\right)$ denotes the Jacobian of $\boldsymbol{g}_{d}\left(\boldsymbol{q}_{n}\right)$ and $D_{\alpha} L_{d}$ denotes the derivative of the discrete Lagrangian with respect to the $\alpha$ th variable. Due to the variational derivation of this scheme, the discrete trajectory conserves a discrete symplectic form and is consistent in momentum maps, i.e., any change in the value of a momentum map reflects exactly the applied forces [11]. Furthermore, the solution shows "good energy behavior" in the sense that energy is not gained or dissipated numerically, which is typical for symplectic methods [12].

4.1.2 The Discrete Null Space Method. To eliminate the discrete constraint forces from the equations, a discrete null space matrix fulfilling range $\left(\boldsymbol{P}\left(\boldsymbol{q}_{n}\right)\right)=\operatorname{null}\left(\boldsymbol{G}_{d}\left(\boldsymbol{q}_{n}\right)\right)$ is employed. Premultiplying $(10)_{1}$ by the transposed discrete null space matrix cancels the constraint forces, i.e., the Lagrange multipliers are eliminated from the set of unknowns, and the system's dimension is reduced to $n$.

4.1.3 Nodal Reparameterization. A reduction in the system to the minimal possible dimension can be accomplished by a local reparameterization of the constraint manifold. At the time nodes, $\boldsymbol{q}_{n}=\boldsymbol{F}\left(\boldsymbol{u}_{n}, \boldsymbol{q}_{n-1}\right)$ is expressed in terms of the discrete generalized coordinates $\boldsymbol{u}_{n} \in U \subseteq \mathbb{R}^{n-m}$ by the map $\boldsymbol{F}: U \subseteq \mathbb{R}^{n-m} \times Q \rightarrow C$, such that the constraints are fulfilled. The discrete generalized control forces are assumed to be constant in each time interval (see Fig. 1). First of all, the effect of the generalized forces acting in $\left[t_{n-1}, t_{n}\right]$ and $\left[t_{n}, t_{n+1}\right]$ is transformed to the time node $t_{n}$ via $\tau_{n-1}^{+}$ $=(h / 2) \tau_{n-1}$ and $\tau_{n}^{-}=(h / 2) \tau_{n}$. Second, the components of the discrete force vectors $\boldsymbol{f}_{n-1}^{+}, f_{n}^{-} \in T_{\boldsymbol{q}_{n}}^{*} Q$ can be calculated as 
$\tau$

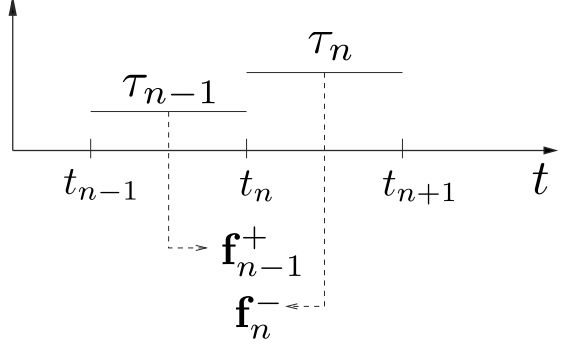

Fig. 1 Relation of redundant forces $f_{n-1}^{+}, f_{n}^{-}$at $t_{n}$ to piecewise constant discrete generalized forces $\tau_{n-1}, \tau_{n}$

$$
\boldsymbol{f}_{n-1}^{+}=\boldsymbol{B}^{T}\left(\boldsymbol{q}_{n}\right) \boldsymbol{\tau}_{n-1}^{+} \quad \boldsymbol{f}_{n}^{-}=\boldsymbol{B}^{T}\left(\boldsymbol{q}_{n}\right) \boldsymbol{\tau}_{n}^{-}
$$

with the $n \times(n-m)$ configuration dependent input transformation matrix $\boldsymbol{B}^{T}: T^{*} U \rightarrow T^{*} Q$.

Upon insertion of the nodal reparameterization, the resulting scheme

$$
\boldsymbol{P}^{T}\left(\boldsymbol{q}_{n}\right)\left[D_{2} L_{d}\left(\boldsymbol{q}_{n-1}, \boldsymbol{q}_{n}\right)+D_{1} L_{d}\left(\boldsymbol{q}_{n}, \boldsymbol{F}\left(\boldsymbol{u}_{n+1}, \boldsymbol{q}_{n}\right)\right)+\boldsymbol{f}_{n-1}^{+}+\boldsymbol{f}_{n}^{-}\right]=\mathbf{0}
$$

has to be solved for $\boldsymbol{u}_{n+1}$, where $\boldsymbol{q}_{n+1}$ is obtained from local reparameterization $\boldsymbol{F}$ of the constraint manifold. Note that the locality of this reparameterization avoids the danger of singularities, which is present in formulations that start with a Lagrangian in generalized coordinates. The reduced scheme (11)) is equivalent to the constrained scheme (10); thus, it also has the key properties of exact constraint fulfillment, symplecticity, and momentum consistency. While the constrained scheme (10) becomes increasingly ill-conditioned for decreasing time-steps, the condition number of Eq. (11) is independent of the time-step.

4.1.4 Boundary Conditions. In the next step, the boundary conditions $\boldsymbol{q}\left(t_{0}\right)=\boldsymbol{q}^{0}$ and $\dot{\boldsymbol{q}}\left(t_{0}\right)=\dot{\boldsymbol{q}}^{0}$, and $\boldsymbol{q}\left(t_{N}\right)=\boldsymbol{q}^{N}$ and $\dot{\boldsymbol{q}}\left(t_{N}\right)=\dot{\boldsymbol{q}}^{N}$ are formulated in the discrete setting. Let $\boldsymbol{q}_{00} \in C$ be a fixed reference configuration, relative to which the initial configuration is computed as $\boldsymbol{q}_{0}=\boldsymbol{F}\left(\boldsymbol{u}_{0}, \boldsymbol{q}_{00}\right)$. To prescribe an initial configuration at $t_{0}$, one can request $\boldsymbol{u}_{0}=\boldsymbol{u}^{0}$. However, since the relative reparameterization computes for $\boldsymbol{q}_{N}$ in terms of $\boldsymbol{u}_{N}$ and $\boldsymbol{q}_{N-1}$, prescribing $\boldsymbol{u}_{N}$ does not enforce a unique final configuration. Final configuration conditions have to be formulated in terms of $\boldsymbol{q}^{N}$ depending on the specific system under consideration (see Sec. 4.2.1 for an example). Since in the present formulation of constrained forced discrete variational dynamics on $Q \times Q$, wherein velocities are not properly defined, velocity conditions have to be transformed into conditions on the conjugate momentum, which are defined at each and every time node using a discrete Legendre transform. Three different discrete Legendre transforms have been defined in Ref. [5]. The reduced discrete Legendre transforms are the most appropriate version to formulate the boundary conditions on the momentum level as

$$
\begin{gathered}
\boldsymbol{P}^{T}\left(\boldsymbol{q}_{0}\right)\left[D_{2} L\left(\boldsymbol{q}^{0}, \dot{\boldsymbol{q}}^{0}\right)+D_{1} L_{d}\left(\boldsymbol{q}_{0}, \boldsymbol{q}_{1}\right)+\boldsymbol{f}_{0}^{-}\right]=\mathbf{0} \\
\boldsymbol{P}^{T}\left(\boldsymbol{q}_{N}\right)\left[D_{2} L\left(\boldsymbol{q}^{N}, \dot{\boldsymbol{q}}^{N}\right)-D_{2} L_{d}\left(\boldsymbol{q}_{N-1}, \boldsymbol{q}_{N}\right)-\boldsymbol{f}_{N-1}^{+}\right]=\mathbf{0}
\end{gathered}
$$

Here, the continuous Legendre transforms $\boldsymbol{p}^{0}=D_{2} L\left(\boldsymbol{q}^{0}, \dot{\boldsymbol{q}}^{0}\right)$ and $\boldsymbol{p}^{N}=D_{2} L\left(\boldsymbol{q}^{N}, \dot{\boldsymbol{q}}^{N}\right)$ are applied to the prescribed boundary velocities.

4.1.5 The Discrete Constrained Optimization Problem. To formulate the optimal control problem for the constrained discrete motion, an approximation

$$
J_{d}\left(\boldsymbol{u}_{d}, \boldsymbol{\tau}_{d}\right)=\sum_{n=0}^{N-1} B_{d}\left(\boldsymbol{u}_{n}, \boldsymbol{u}_{n+1}, \boldsymbol{\tau}_{n}\right)
$$

of the continuous objective functional $J(\boldsymbol{q}, \dot{\boldsymbol{q}}, \boldsymbol{f})=\int_{t_{0}}^{t_{N}} B(\boldsymbol{q}, \dot{\boldsymbol{q}}, \boldsymbol{f}) d t$ has to be defined, where $B(\boldsymbol{q}, \dot{\boldsymbol{q}}, \boldsymbol{f}): T C \times T^{*} Q \rightarrow \mathbb{R}$ is a given cost function. The objective function (13) has to be minimized with respect to $\boldsymbol{u}_{d}=\left\{\boldsymbol{u}_{n}\right\}_{n=0}^{N}$ and $\boldsymbol{\tau}_{d}=\left\{\boldsymbol{\tau}_{n}\right\}_{n=0}^{N-1}$ subject to a minimal set of

(a)
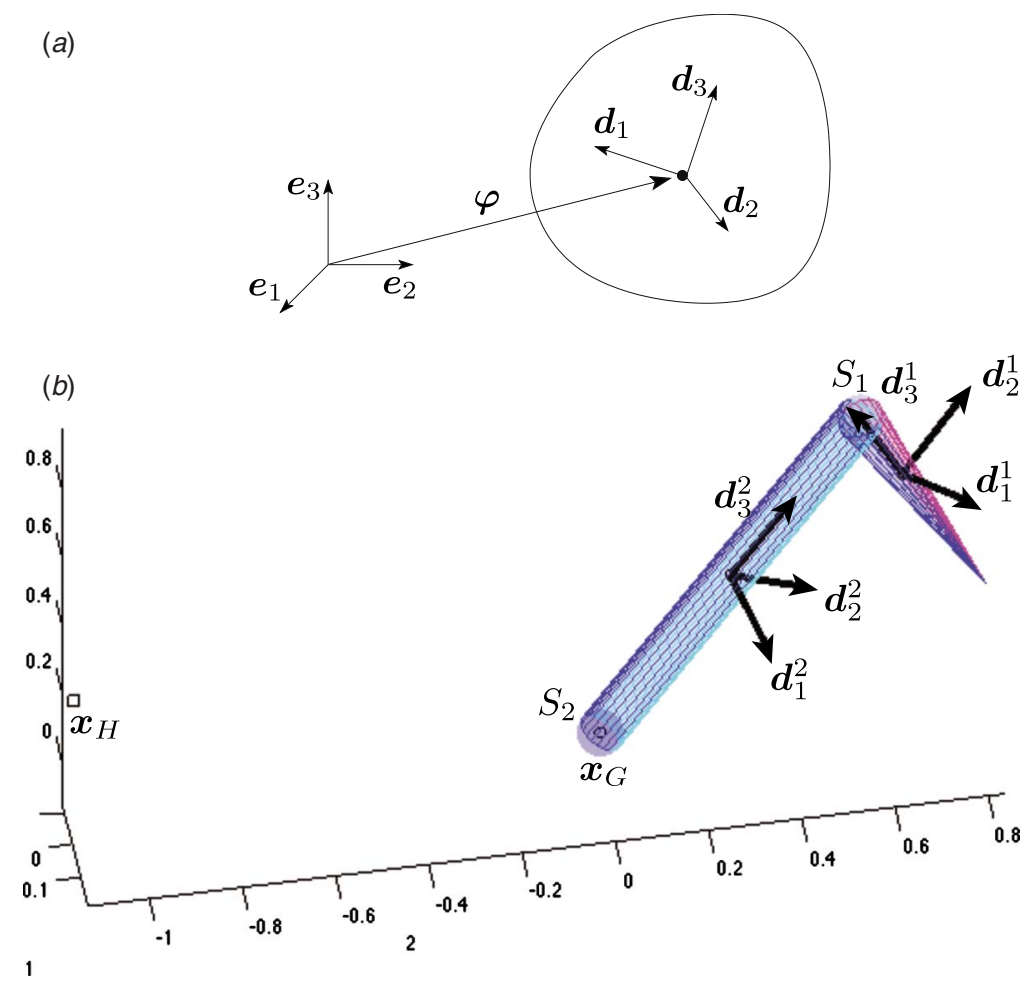

Fig. 2 Configuration of a rigid body (a) and initial configuration of the robot arm consisting of two rigid bodies combined into a spherical pair by the joint $S_{1}$ and fixed in space by the spherical joint $S_{2}(b)$ 

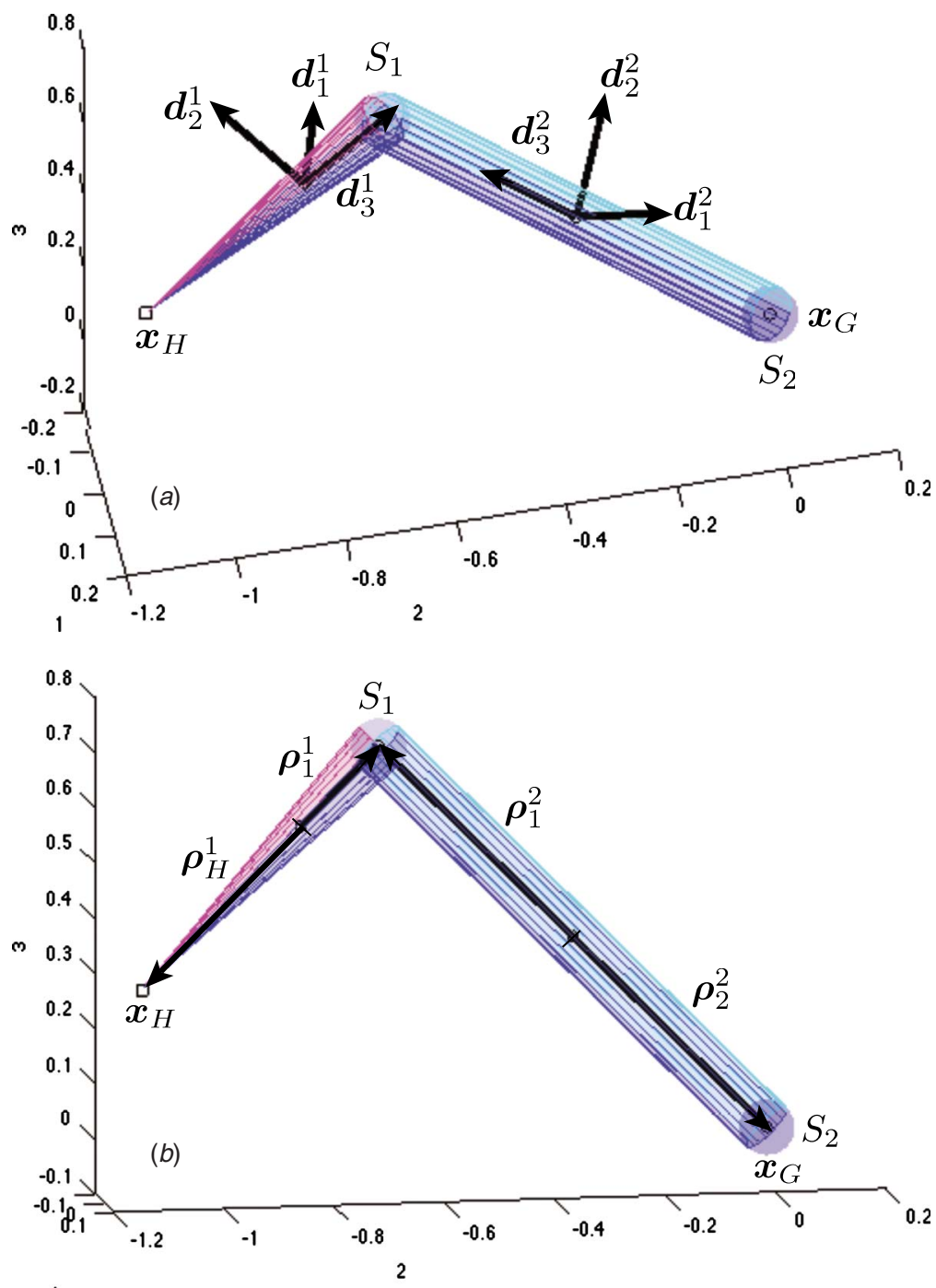

Fig. 3 Final configuration of the robot arm showing the director triads $\left\{d_{l}^{\alpha}\right\}$ $(\alpha=1,2, I=1,2,3)(a)$ and the joint location vectors $\rho_{\beta}^{\alpha}(\beta=1,2)(b)$

initial and final configuration constraints, initial and final momentum constraints (Eq. (12)), and discrete equations of motion (Eq. (11)) for $n=1, \ldots, N-1$. Furthermore, time-dependent path, constraints prescribing (parts of) the motion, and inequality constraints bounding the optimization variables can be present.

Remark 4.1 (Dimension of the constrained optimization problem). The use of the discrete null space method with nodal reparameterization yields a constrained optimization problem of minimal possible dimension: the optimization of Eq. (13) subject to the boundary conditions and Eq. (11) includes the $(2 N+1)(n$ $-m$ ) variables $\boldsymbol{u}_{d}, \boldsymbol{\tau}_{d}$ and $(N+3)(n-m)$ constraints. In contrast to that, the constrained optimization problem resulting from the Lagrange multiplier formulation (Eq. (10)) involves the $N(2 n$ $+m)+n$ unknowns $\boldsymbol{q}_{d}, \boldsymbol{f}_{d}, \boldsymbol{\lambda}_{d}$ and $(N-1)(n+m)+4 n$ constraints (this are $(3 N+1) m$ more variables and $(N+1) 2 m$ more constraints). Of course, this influences the computational costs and the spectrum of available methods to solve the problem substantially.

4.2 Deterministic Optimal Control of the Robot Arm. This section describes the constrained formulation of the cranelike robot arm and its optimal control using DMOCC to compute for the optimal trajectory and control sequence steering the arm from its initial position, as depicted in Fig. 2 (right), to the final position, where the tip is located in $\boldsymbol{x}_{H}$, as shown in Fig. 3. The objective of this rest to rest maneuver is the minimization of the control effort $J_{d}\left(\boldsymbol{u}_{d}, \boldsymbol{\tau}_{d}\right)=\sum_{n=0}^{N-1}\left\|\boldsymbol{\tau}_{n}\right\|^{2}$. The robot-arm model consists of two rigid bodies and two spherical joint connections, the first body being a cone and the second body being a cylinder (see Fig. 2, right). The first spherical joint $S_{1}$ connects the two bodies by preventing relative translation. However, relative rotation of the bodies is not constrained. The second joint $S_{2}$ fixes the end of the cylinder in space at $\boldsymbol{x}_{G}$.

In contrast to rotation based approaches to rigid body dynamics taken from Refs. $[13,14]$, here, each rigid body is viewed as a constrained continuum, which is described in redundant coordinates subject to holonomic constraints $[15,16]$. The $\alpha$ th rigid body's configuration variable

$$
\boldsymbol{q}^{\alpha}=\left[\begin{array}{c}
\boldsymbol{\varphi}^{\alpha} \\
\boldsymbol{d}_{1}^{\alpha} \\
\boldsymbol{d}_{2}^{\alpha} \\
\boldsymbol{d}_{3}^{\alpha}
\end{array}\right] \in \mathbb{R}^{12} \quad \alpha=1,2
$$

consists of the placement of the center of mass $\varphi^{\alpha} \in \mathbb{R}^{3}$ and the directors $\boldsymbol{d}_{I}^{\alpha} \in \mathbb{R}^{3}(I=1,2,3)$, which are constrained to stay orthonormal during the motion (see Figs. 2 and 3, left). The equations of motion assume the form of DAEs with a constant mass matrix. This formulation circumvents many difficulties associated with 
rotational parameters $[17,18]$ and can be generalized easily to three-dimensional multibody systems consisting of many rigid bodies and also elastic structural elements $[19,20]$. The location of the $\beta$ th joint in the $\alpha$ th body is characterized by coordinates $\left(\rho_{\beta}^{\alpha}\right)_{i}$ in the body frame $\left\{\boldsymbol{d}_{I}^{\alpha}\right\}$, where $\alpha, \beta=1,2$ (see Fig. 3, right)

$$
\boldsymbol{\rho}_{\beta}^{\alpha}=\left(\rho_{\beta}^{\alpha}\right)_{i} \boldsymbol{d}_{i}^{\alpha}
$$

4.2.1 Null Space Matrix. The null space matrix associated with the robot arm is given by

$$
\boldsymbol{P}(\boldsymbol{q})=\left[\begin{array}{cc}
\widehat{\boldsymbol{\rho}_{1}^{1}} & \widehat{\boldsymbol{\rho}_{2}^{2}}-\widehat{\boldsymbol{\rho}_{2}^{1}} \\
-\widehat{\mathbf{d}_{1}^{1}} & \mathbf{0} \\
-\widehat{\boldsymbol{d}_{2}^{1}} & \mathbf{0} \\
-\widehat{\boldsymbol{d}_{3}^{1}} & \mathbf{0} \\
\mathbf{0} & \widehat{\boldsymbol{\rho}_{2}^{2}} \\
\mathbf{0} & -\widehat{\boldsymbol{d}}_{1}^{2} \\
\mathbf{0} & -\widehat{\boldsymbol{d}}_{2}^{2} \\
\mathbf{0} & -\widehat{\boldsymbol{d}}_{3}^{2}
\end{array}\right]
$$

with the hat map $\hat{\imath} \mathbb{R}^{3} \rightarrow \operatorname{so}(3)$ and $\mathbf{0}$ denoting the $3 \times 3$ zero matrix.

4.2.2 Nodal Reparameterization. Let $\boldsymbol{\theta}_{n+1}^{1}$ and $\boldsymbol{\theta}_{n+1}^{2}$ represent the incremental rotation vectors pertaining to the two bodies. In particular, the nodal reparameterization reads

$$
\boldsymbol{q}_{n+1}=\left[\begin{array}{c}
\boldsymbol{x}_{G}+\exp \left(\widehat{\boldsymbol{\theta}_{n+1}^{2}}\right)\left(-\left(\boldsymbol{\varrho}_{2}^{2}\right)_{n}+\left(\boldsymbol{\varrho}_{1}^{2}\right)_{n}\right)-\exp \left(\widehat{\boldsymbol{\theta}_{n+1}^{1}}\right)\left(\boldsymbol{\varrho}_{1}^{1}\right)_{n} \\
\exp \left(\widehat{\boldsymbol{\theta}_{n+1}^{1}}\right)\left(\boldsymbol{d}_{1}^{1}\right)_{n} \\
\exp \left(\widehat{\boldsymbol{\theta}_{n+1}^{1}}\right)\left(\boldsymbol{d}_{2}^{1}\right)_{n} \\
\exp \left(\widehat{\boldsymbol{\theta}_{n+1}^{1}}\right)\left(\boldsymbol{d}_{3}^{1}\right)_{n} \\
\boldsymbol{x}_{G}-\exp \left(\widehat{\boldsymbol{\theta}_{n+1}^{2}}\right)\left(\boldsymbol{\varrho}_{2}^{2}\right)_{n} \\
\exp \left(\widehat{\boldsymbol{\theta}_{n+1}^{2}}\right)\left(\boldsymbol{d}_{1}^{2}\right)_{n} \\
\exp \left(\widehat{\boldsymbol{\theta}_{n+1}^{2}}\right)\left(\boldsymbol{d}_{2}^{2}\right)_{n} \\
\exp \left(\widehat{\boldsymbol{\theta}_{n+1}^{2}}\right)\left(\boldsymbol{d}_{3}^{2}\right)_{n}
\end{array}\right]
$$

Rodrigues' formula is used to obtain a closed form expression of the exponential map exp:so(3) $\rightarrow S O(3)$, mapping skewsymmetric matrices to proper rotations [21].

4.2.3 Actuation of the Robot Arm. The actuation of the arm is twofold. First of all, the spherical joint connection $S_{1}$ is actuated by the joint torque $\boldsymbol{\tau}_{\theta_{1}} \in \mathbb{R}^{3}$. It affects both bodies, where according to "action equals reaction," the resulting generalized forces on the bodies are equal, but opposite in sign [22]. Second, the torque $\boldsymbol{\tau}_{\theta_{2}} \in \mathbb{R}^{3}$ actuates $S_{2}$ and affects the second body only.

The redundant forces on the bodies' center of mass and the directors corresponding to the configuration variable (Eq. (14)) can then be computed as

$$
\boldsymbol{f}=\left[\begin{array}{l}
\boldsymbol{f}^{1} \\
\boldsymbol{f}^{2}
\end{array}\right]=\boldsymbol{B}^{T}(\boldsymbol{q})\left[\begin{array}{l}
\boldsymbol{\tau}_{\theta_{1}} \\
\boldsymbol{\tau}_{\theta_{2}}
\end{array}\right]
$$

with the $24 \times 6$ input transformation matrix

$$
\boldsymbol{B}^{T}(\boldsymbol{q})=\left[\begin{array}{cc}
\mathbf{0} & \mathbf{0} \\
\frac{1}{2} \widehat{\boldsymbol{d}_{1}^{1}} & \mathbf{0} \\
\frac{1}{2} \widehat{\boldsymbol{d}_{2}^{1}} & \mathbf{0} \\
\frac{1}{2} \widehat{\boldsymbol{d}}_{3}^{1} & \mathbf{0} \\
\mathbf{0} & \mathbf{0} \\
-\frac{1}{2} \widehat{\boldsymbol{d}}_{1}^{2} & \frac{1}{2} \widehat{\boldsymbol{d}_{1}^{2}} \\
-\frac{1}{2} \widehat{\boldsymbol{d}_{2}^{2}} & \frac{1}{2} \widehat{\boldsymbol{d}_{2}^{2}} \\
-\frac{1}{2} \widehat{\boldsymbol{d}_{3}^{2}} & \frac{1}{2} \widehat{\boldsymbol{d}_{3}^{2}}
\end{array}\right]
$$

Angular momentum of the robot arm reads $\boldsymbol{L}=\stackrel{2}{\Sigma}^{2} \boldsymbol{\varphi}^{\alpha} \times \boldsymbol{p}_{\boldsymbol{\varphi}}^{\alpha}+\boldsymbol{d}_{I}^{\alpha}$ $\alpha=1$

$\times \boldsymbol{p}_{I}^{\alpha}$, where the summation convention is used to sum over the repeated index $I$. In the discrete setting, the angular momentum at $t_{n}$ can be computed in terms of the conjugate momenta obtained via the constrained discrete Legendre transform, or equivalently, via the projected discrete Legendre transform [5].

In the present case of the robot arm, the change in angular momentum is induced by $\boldsymbol{\tau}_{\theta_{2}}$ and the force due to the gravitational potential

$$
V(\boldsymbol{q})=\left[00-M_{\varphi}^{1} g \mathbf{0}_{1 \times 9} 00 M_{\varphi}^{2} g \mathbf{0}_{1 \times 9}\right] \boldsymbol{q}
$$

with the acceleration $g \in \mathbb{R}$ and the total masses $M_{\varphi}^{\alpha}(\alpha=1,2)$ of the bodies. In particular, the change in angular momentum in one time interval is given by

$$
\begin{aligned}
\boldsymbol{L}_{n+1}-\boldsymbol{L}_{n}= & \left(\boldsymbol{\tau}_{\theta_{2}}\right)_{n}^{+}+\left(\boldsymbol{\tau}_{\theta_{2}}\right)_{n}^{-}-\left(\boldsymbol{\varphi}_{n+1}^{1}+\boldsymbol{\varphi}_{n}^{1}\right) \times \frac{h}{2}\left[00-M_{\varphi}^{1} g\right]-\left(\boldsymbol{\varphi}_{n+1}^{2}\right. \\
& \left.+\boldsymbol{\varphi}_{n}^{2}\right) \times \frac{h}{2}\left[00-M_{\varphi}^{2} g\right]
\end{aligned}
$$

The consistency of the momentum maps can be proven analytically and is illustrated numerically in Fig. 4.

4.2.4 Robot-Arm Maneuver in the Deterministic Setting. The particular robot arm we consider consists of a cone of radius $r^{1}$ $=0.05$ length $l^{1}=0.6$, and mass $M_{\varphi}^{1}=10$, and a cylinder of radius $r^{2}=0.05$, length $l^{2}=0.5$, and mass $M_{\varphi}^{2}=5$. One end of the cylinder is fixed in space at $x_{G}=[000]$ by the spherical joint $S_{2}$, while the other end is coupled to the cylinder via $S_{2}$. In the reference configuration $\boldsymbol{q}_{00}$ depicted in Fig. 2 (right), both bodies are tilted from a vertical position by a rotation of $\pi / 4$ around the axis $\boldsymbol{e}_{1}$. The directors are aligned with the bodies' principal axes of inertia such that $\boldsymbol{d}_{3}^{\alpha}$ coincides with the longitudinal axis.

At the start of the maneuver, the initial configuration must coincide with the reference configuration; thus, $\boldsymbol{q}_{0}=\boldsymbol{q}_{00}$ and the initial configuration condition for the optimal control problem reads $\boldsymbol{u}_{0}=\mathbf{0}_{6 \times 1}$. In the final configuration, the tip of the cone must coincide with a prescribed location $\boldsymbol{x}_{H}=\left[\begin{array}{lll}0-1.131 & 0.283\end{array}\right]$ in space. Using the vector $\boldsymbol{\rho}_{H}^{1}$ specifying the location of the tip in the body frame $\left\{\boldsymbol{d}_{I}^{1}\right\}$ (see Fig. 3 , right), the final configuration condition reads $\boldsymbol{\varphi}_{N}^{1}+\left(\boldsymbol{\rho}_{H}^{1}\right)_{N}-\boldsymbol{x}_{H}=\mathbf{0}_{3 \times 1}$. The desired motion is a rest to rest maneuver; thus, $\boldsymbol{p}^{0}=\boldsymbol{p}^{N}=\mathbf{0}_{24 \times 1}$ in the boundary conditions on the momentum level (Eq. (12)). In the deterministic setting, the objective function (13) represents the control effort; therefore, the convex objective function $J_{d}\left(\boldsymbol{u}_{d}, \boldsymbol{\tau}_{d}\right)=\sum_{n=0}^{N-1}\left\|\boldsymbol{\tau}_{n}\right\|^{2}$ is minimized subject to the described boundary conditions and the discrete equations of motion (Eq. (11)). Furthermore, bound constraints insure 

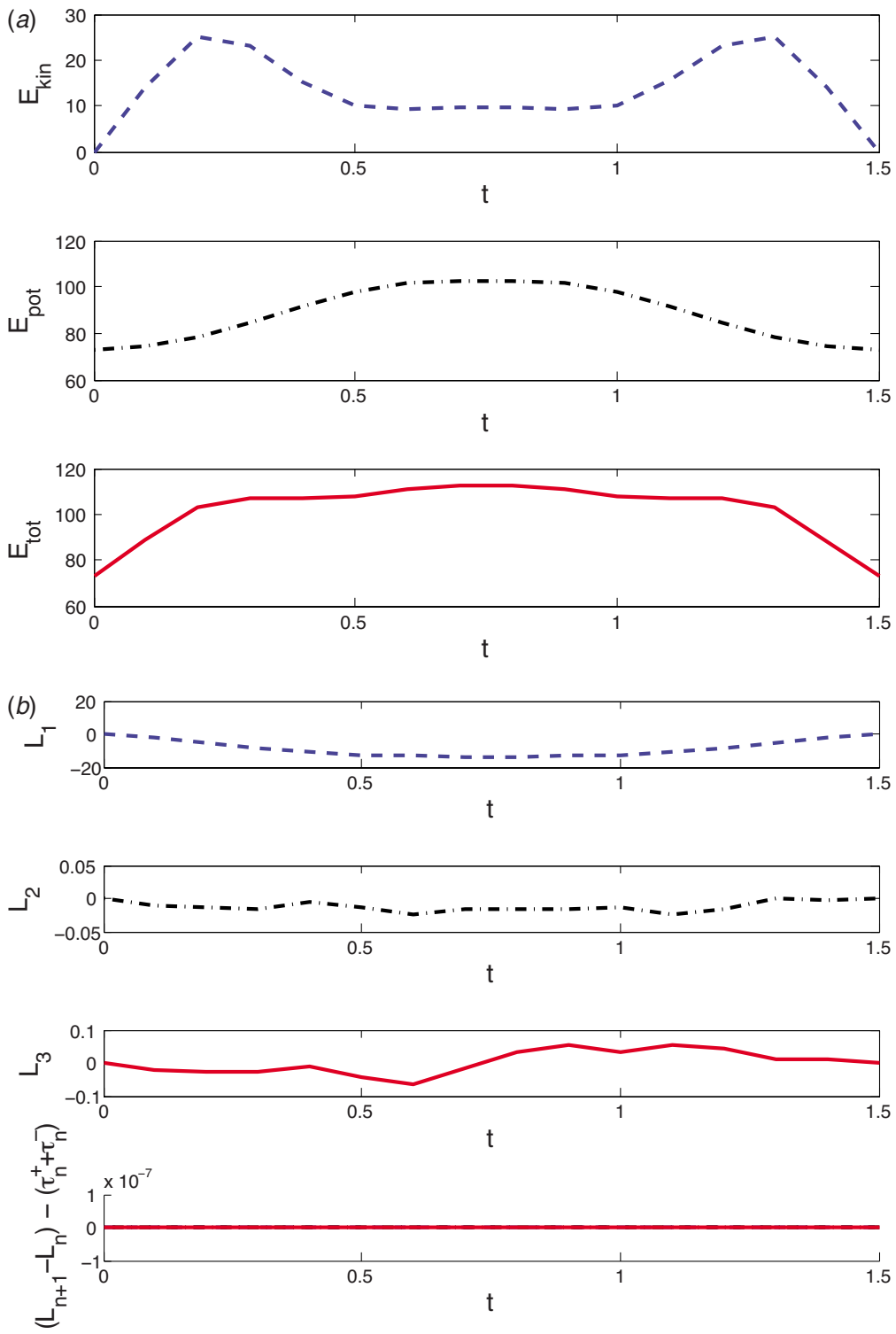

Fig. 4 Evolution of the kinetic, potential, and total energies (a) and components of the angular momentum $(b)$. The bottom plot on the right shows that the momentum maps are represented consistently.

that the components of the applied torques are in the range of $-120 \leq\left(\left(\tau_{\theta_{\alpha}}\right)_{i}\right)_{n} \leq 120$, where $\alpha=1,2, i=1,2,3$ and $n=0, N-1$. The maneuver takes place in $t_{N}=1.5$, and $N=15$ time-steps of size $h=0.1$ are used.

The left hand plot in Fig. 4 shows the evolution of the kinetic, potential, and total energies. In particular, the first plot illustrates that the maneuver starts and ends at rest. The evolution of the components of angular momentum is shown on the right hand side. The bottom plot verifies Eq. (15) numerically (note that $\left(\boldsymbol{\tau}_{n}^{+}+\boldsymbol{\tau}_{n}^{-}\right)$in the plot represents the entire right hand side of Eq. (15)). The evolution of the components of the torques in each joint can be observed from Fig. 5 (left). As described before, the torques are constant in each time interval. Finally, the resulting tip trajectory is depicted in Fig. 5 on the right hand side.

Remark 4.2 (Implementation). The constrained minimization has been performed by the SQP solver FMINCON in MATLAB, which can handle bound constraints on the optimization variables as well as linear and nonlinear equality and inequality constraints. The gradient of the objective function and the Jacobian of the nonlinear equality constraints have been derived analytically, and are given as user-supplied derivatives to MATLAB. This substantially reduces the computational costs compared with the case when MATLAB approximates the derivatives via finite differencing.

Remark 4.3 (Inequality constraints). No inequality constraints have been imposed on the minimization in this simulation. To obtain more realistic maneuvers, it is necessary to prevent interpenetration of the two bodies via appropriate inequality constraints. This is left for future work.

\section{Test Case: Minimizing the Probability of Failure for a Robot-Arm Maneuver}

In this section, the deterministic robot-arm maneuver from Sec. 4.2.1 is reconsidered in the presence of uncertainty. Specifically, we consider two different uncertainty cases. First, there is uncertainty in the geometry of the robot arm, i.e., the lengths $l^{1}$ and $l^{2}$ are uncertain. Second, uncertain operating conditions are represented by the presence of uncertain wind forces in addition to the uncertain lengths. In all calculations we use the reduced variational time-stepping scheme (11) obtained via the discrete null 


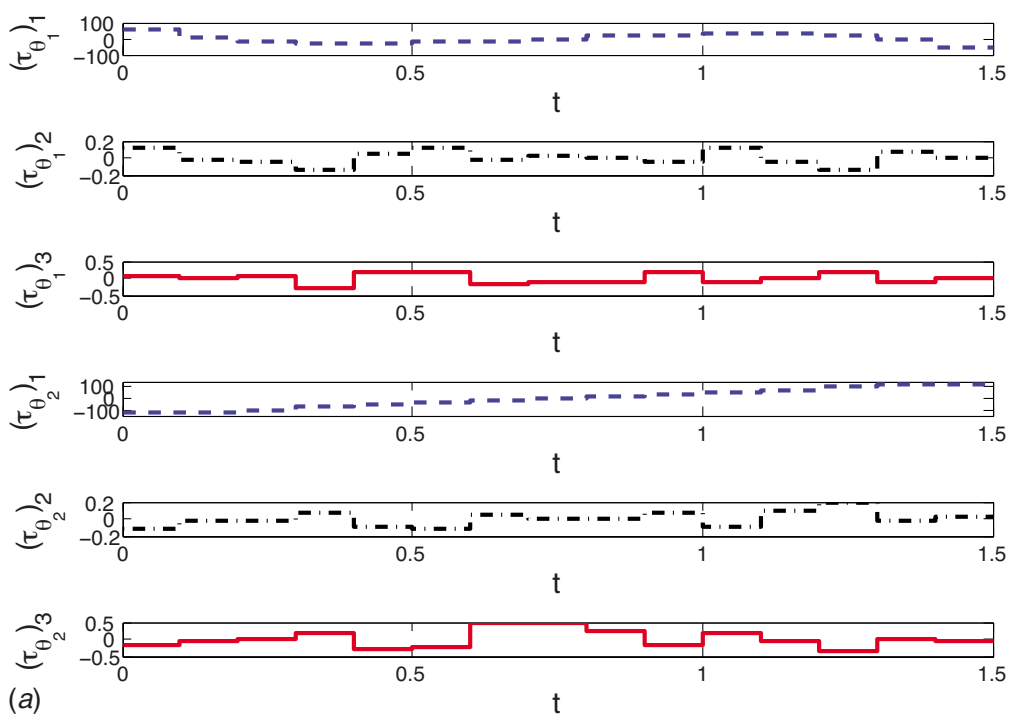

(a)

$t$

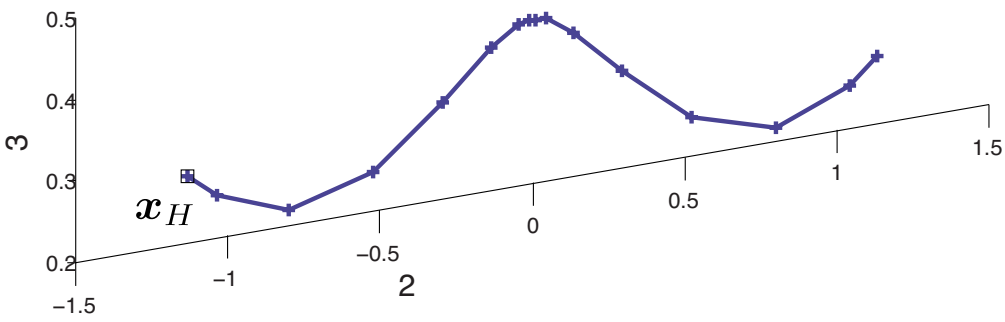

1

(b)

Fig. 5 Evolution of the torques (a) and trajectory of the tip (b)

space method with nodal reparameterization. By design, this scheme is symplectic and represents changes in the momentum maps exactly. Furthermore, energy is not gained or dissipated numerically. As it is typical for the integration of constrained dynamics, the discrete equations of motion are implicit and need to be solved iteratively, e.g., by means of a Newton-Raphson iteration.

The performance measure $Y$ of interest is assumed to be the placement accuracy of the arm tip, i.e., the distance from the arm tip and its prescribed location $\boldsymbol{x}_{H}$ at the end of the maneuver's duration of $t_{N}=1.5$. Thus, in this case $Y^{\tau_{d}}=\left\|\boldsymbol{\varphi}_{N}^{1}+\left(\boldsymbol{\rho}_{H}^{1}\right)_{N}-\boldsymbol{x}_{H}\right\|$ is obtained for a candidate control sequence $\tau_{d}$ by stepping forward in time using Eq. (11) and the initial conditions described in Sec. 4.2.1. From an optimal control point of view, this is similar to a shooting approach to solve the concentration-of-measure optimal control problem (Eq. (9)). We additionally suppose that a positioning accuracy $a$ is prescribed, so that the robot arm operates safely if $Y \leq a$ and fails if $Y>a$. The goal is to find a control sequence $\tau_{d}$ for which the confidence in safe operation is maximal, i.e., the resulting objective function to be minimized is

$$
-\mathrm{CF}\left(\boldsymbol{\tau}_{d}\right) \equiv-\frac{M^{\tau_{d}}}{U^{\tau_{d}}} \equiv-\frac{\left(a-\left\langle Y^{\tau_{d}}\right\rangle-\alpha^{\tau_{d}}\right)_{+}}{D_{F_{d}}}
$$

The evaluation of the objective function (16) requires the evaluation of the mean response and the diameter. In all calculations presented here, the mean response (Eq. (5)) is computed by random Monte Carlo sampling, and the system diameter (Eq. (3)) and optimal controls $\tau_{d}$ are computed by simulated annealing. The basic simulated-annealing algorithm is that described in Ref. [23] and has been enhanced with several user-specified options to suit our needs. Details may be found in the Appendix. The starting controls for the iteration are set to the deterministic controls computed in Sec. 4.2.1.

5.1 Uncertain Geometry. First, there are $M=2$ uncertain variables. Length $l^{1}$ can vary randomly in a range of $5 \%$ and $l^{2}$ varies randomly in the range of $0.1 \%$ around the given value. These values assure that their influence on the system's uncertainty is of the same order of magnitude.

The evolution of the mean performance, system diameter, and concentration-of-measure probability of failure upper bound along the simulated-annealing iteration for the determination of the optimal controls is shown in Fig. 6. As expected, both the positioning accuracy of the maneuver, which is measured by the mean response $\langle Y\rangle$ with $m=100$, and the uncertainty in the operation of the maneuver, which is measured by the diameter $D_{F}$, show a decreasing tendency. Correspondingly, the concentration-ofmeasure probability of failure upper bound decreases from initially $\mathrm{P}=0.49$ to $\mathrm{P}^{\text {best }}=0.013722$. This reduction in the probability of failure may be alternatively interpreted as an increase in the confidence that may be placed in the safe operation of the maneuver, as measured by the confidence factor (Eq. (7)). Recall that the right hand side of Eq. (6) is a random variable, and with probability at most $\epsilon^{\prime}$, it may fail to be an upper bound on the probability of failure. This is why, for the optimal control sequence with $\mathbb{P}_{\text {best }}=0.013722$ computed via Eq. (6) (using the empirical mean, which is subject to large deviations as rare events), the mean has been recomputed with $m=10000$. Assuming that the latter empirical mean is an "accurate" approximation of the exact mean in Eq. (4) results in the even lower probability of failure bound $\mathbb{P}_{\text {best }}$ $=0.00047207$. 

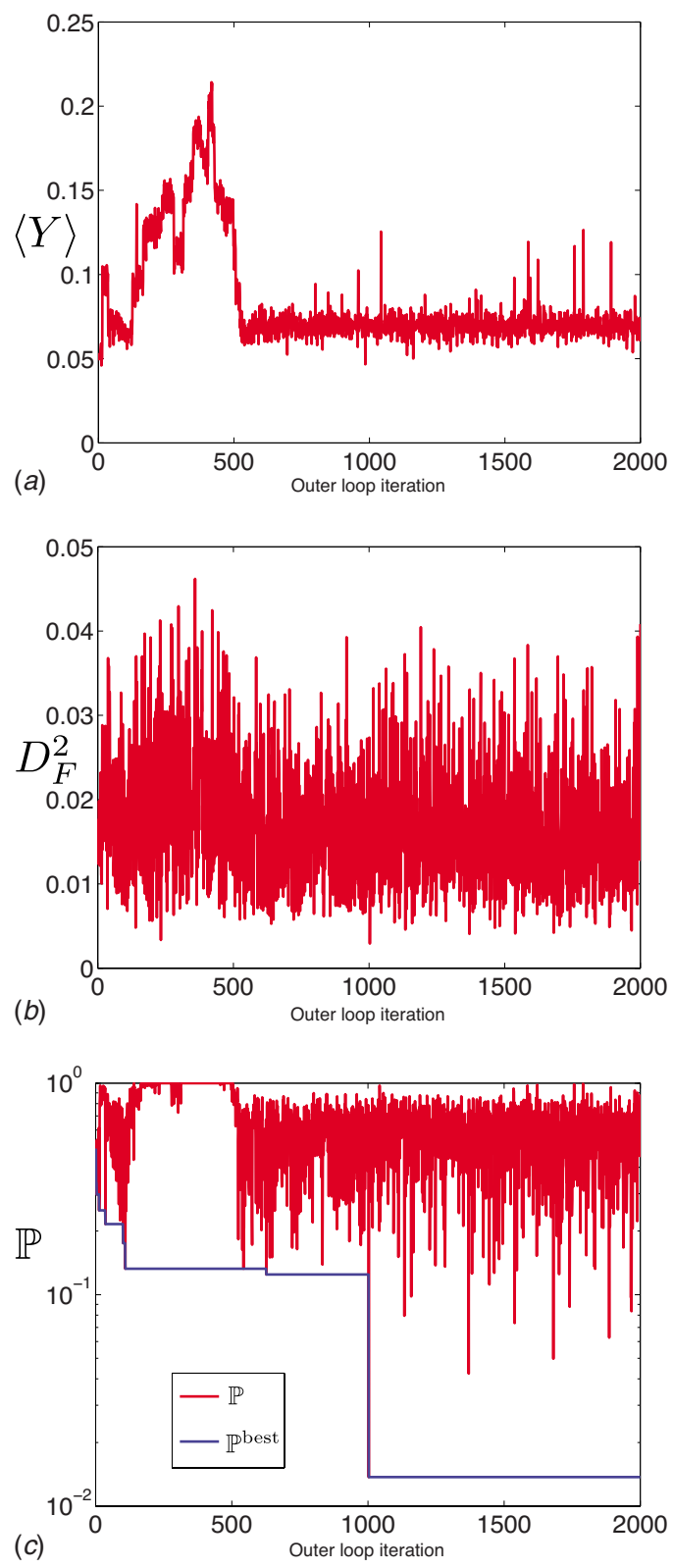

Fig. 6 Uncertain geometry: simulated-annealing iteration for the determination of the optimal controls; evolution of the (a) mean performance, $(b)$ system diameter, and (c) concentrationof-measure probability of failure upper bound

It bears emphasis that high confidence in the safe operation of a system requires achieving a large design margin and a low uncertainty simultaneously. More precisely, confidence requires that the design margin be large in relation to the uncertainty in the operation of the system, which underscores the importance of quantifying - and mitigating by means of optimal controlsystem uncertainties for purposes of certification. Here, again, the ability of COMOC to increase the design confidence in the particular example of the robot-arm maneuver becomes obvious.

5.2 Uncertain Wind Forces and Geometry. Second, in addition to the uncertain lengths, each body is affected by a random wind force in every time-step, hitting the body's surface around the center of mass in a prescribed location. Each component of the two three-dimensional force vectors varies randomly between in $[-0.001,0.001]$. Altogether, $M=92$ uncertain variables are present in this simulation. Figure 7 shows the evolution of the mean performance with $m=100$, system diameter, and
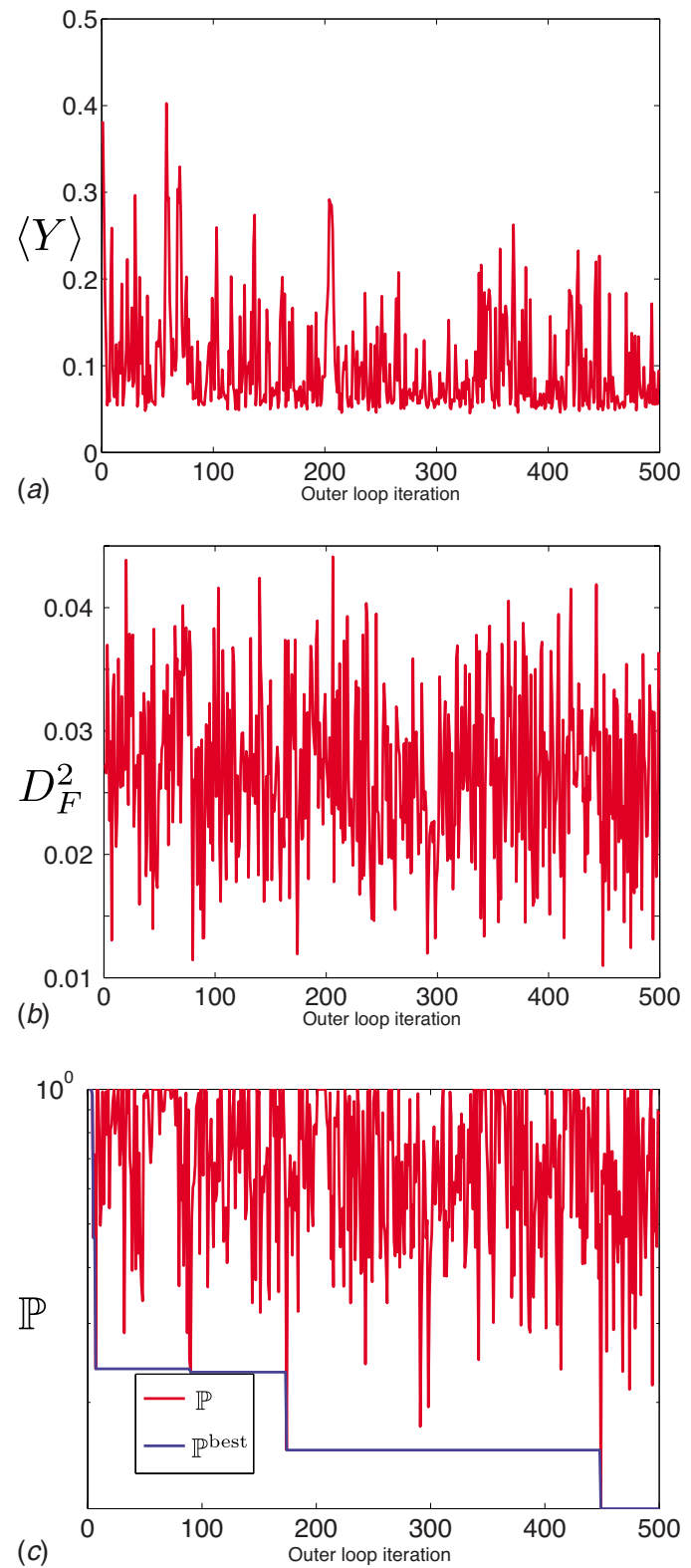

Fig. 7 Uncertain wind forces and uncertain geometry: simulated-annealing iteration for the determination of the optimal controls; evolution of the (a) mean performance, (b) system diameter, and $(c)$ concentration-of-measure probability of failure upper bound

concentration-of-measure probability of failure upper bound along the simulated-annealing iteration. The probability of failure upper bound has been improved from $\mathbb{P}=1$ to $\mathrm{P}^{\text {best }}=0.11581$. Also, here, it can be observed that the Pbest has been found for a control sequence that leads to (local) minima in the mean and diameter, respectively. Again, assuming that the mean resulting from $m$ $=10000$ samples yields exactly the mean performance, Eq. (4) bounds the probability of failure by $\mathrm{P}^{\text {best }}=0.0567$

\section{Summary and Conclusions}

We have presented an optimal control methodology, which we refer to as COMOC, that seeks to minimize the concentration-ofmeasure upper bound on the probability of failure of a system. The systems under consideration are characterized by a single performance measure that depends on random inputs through a known response function. In addition, the safe operation of the 
system is characterized by a threshold value of the performance measure. For these systems, a concentration-of-measure upper bound on the probability of failure of a system can be formulated in terms of the mean performance measure and a system diameter that measures the uncertainty in the operation of the system. COMOC then seeks to determine optimal controls that maximize the confidence in the safe operation of the system, defined as the ratio of design margin, which is measured by the difference between the mean performance and the design threshold, to the system uncertainty, which is measured by the system diameter. This strategy has been assessed in the case of a robot-arm maneuver for which the performance measure of interest is assumed to be the placement accuracy of the arm tip. The ability of COMOC to increase the design confidence in that particular example of application is remarkable and bodes auspiciously for the approach.

The most severe limitation of the COMOC implementation presented in this paper is its computational expense. Each evaluation of the confidence factor objective function requires the calculation of the mean response and system diameter for a particular control, which, in turn, requires multiple solutions of the equations of motion of the system. In order to reduce the computational expense to a tractable level, in the calculations presented here, the controls have been constrained to remain close to the initial deterministic solution. It is conceivable that further gains in the design confidence could be achieved from an unrestricted control optimization, but the computational resources and infrastructure required for such an optimization are beyond the scope of this paper. In view of these present limitations, the formulation of efficient COMOC implementations that alleviate its computational expense clearly suggests itself as a subject of further research.

\section{Acknowledgment}

The authors gratefully acknowledge the support of the U.S. Department of Energy through Caltech's PSAAP Center for the Predictive Modeling and Simulation of High-Energy Density Dynamic Response of Materials. We would also like to thank Sina Ober-Blöbaum for the helpful discussions, and the reviewers for their valuable suggestions in the paper's organization.

\section{Appendix: Optimization Algorithm}

The basic simulated-annealing algorithm is that described in Ref. [23] and has been enhanced with several user-specified options to suit our needs. Define $T$ as "temperature" and $N$ as the number of function evaluations. We use a default cooling schedule of $T_{\text {new }}=0.8 \times T^{\text {old }}$ with $T_{0}=1.0$. The optimization stops if $T$ $\leq 1.0 \times 10^{-8}, N>N_{\max }=2000$, or $N_{R}>300$, where $N_{R}$ is the number of successive rejected states. Temperature decrease happens if $N_{T}>30$ or $N_{S}>20$, i.e., if 30 function evaluations are made, or if there are 20 successive accepted optimal states found at the current temperature. The Boltzmann constant is set to 1.0.

The bound constraints on the random variables need to be satisfied in the optimization algorithm as well. The neighbor-finding routine intelligently seeks out neighboring states that assert compliance of any permutations of these constraints to find a new neighbor by projecting a randomly generated neighbor into a parameterized point within the constrained design space.

\section{References}

[1] Ledoux, M., 2001, The Concentration of Measure Phenomenon, American Mathematical Society, Providence, RI.

[2] Boucheron, S., Bousquet, O., and Lugosi, G., 2004, "Concentration inequalities," Advanced Lectures in Machine Learning, O. Bousquet, U. V. Luxburg, and G. Rätsch, eds., Springer, New York, pp. 208-240.

[3] Lucas, L., Owhadi, H., and Ortiz, M., 2008, "Rigorous Verification, Validation, Uncertainty Quantification and Certification Through Concentration-ofMeasure Inequalities," Comput. Methods Appl. Mech. Eng., 197, pp. 45914609.

[4] Leyendecker, S., Ober-Blöbaum, S., Marsden, J., and Ortiz, M., 2007, "Discrete Mechanics and Optimal Control for Constrained Multibody Dynamics," Proceedings of the Sixth International Conference on Multibody Systems, Nonlinear Dynamics, and Control, ASME International Design Engineering Technical Conferences, Las Vegas, NV, Sept. 4-7.

[5] Leyendecker, S., Ober-Blöbaum, S., Marsden, J., and Ortiz, M., 2009, "Discrete Mechanics and Optimal Control for Constrained Systems (DMOCC)," Opt. Control Appl. Methods, in press.10.1002/oca.912

[6] Lawrence, C., 2004, "An Introduction to Stochastic Differential Equations Version 1.2," University of California at Berkeley, available online at http:// math.berkely.edu/ evans/SDEcourse.pdf

[7] McDiarmid, C., 1989, "On the Method of Bounded Differences," Surveys in Combinatorics (London Mathematical Society Lecture Note Series), Cambridge University Press, Cambridge, England, Vol. 141, pp. 148-188.

[8] Hoeffding, W., 1963, "Probability Inequalities for Sums of Bounded Random Variables," J. Am. Stat. Assoc., 58, pp. 13-30.

[9] Ober-Blöbaum, S., Junge, O., and Marsden, J., "Discrete Mechanics and Optimal Control: An Analysis," ESAIM: Control Optimisation and Calculus of Variations, in press.

[10] Leyendecker, S., Marsden, J., and Ortiz, M., 2008, "Variational Integrators for Constrained Dynamical Systems," Z. Angew. Math. Mech., 88(9), pp. 677708.

[11] Marsden, J., and West, M., 2001, "Discrete Mechanics and Variational Integrators," Acta Numerica, 10, pp. 357-514.

[12] Hairer, E., Wanner, G., and Lubich, C., 2004, Geometric Numerical Integration: Structure-Preserving Algorithms for Ordinary Differential Equations, Springer, New York.

[13] Krysl, P., 2005, "Direct Time Integration of Rigid Body Motion With DiscreteImpulse Midpoint Approximation: Explicit Newmark Algorithms," Commun. Numer. Methods Eng., 22(5), pp. 441-451.

[14] Bou-Rabee, N., and Marsden, J., 2009, "Hamilton-Pontryagin Integrators on Lie Groups: Introduction and Structure-Preserving Properties," Found Comput. Math., 9(2), pp. 197-219.

[15] Antmann, S., 1995, Nonlinear Problems in Elasticity, Springer, New York.

[16] Reich, S., 1996, "Symplectic Integrators for Systems of Rigid Bodies," Fields Inst. Commun., 10, pp. 181-191.

[17] Betsch, P., Menzel, A., and Stein, E., 1998, "On the Parametrization of Finite Rotations in Computational Mechanics: A Classification of Concepts With Application to Smooth Shells," Comput. Methods Appl. Mech. Eng., 155, pp. 273-305.

[18] Bauchau, O., and Trainelli, L., 2003, “The Vectorial Parameterization of Rotation," Nonlinear Dyn., 32(1), pp. 71-92.

[19] Betsch, P., and Leyendecker, S., 2006, "The Discrete Null Space Method for the Energy Consistent Integration of Constrained Mechanical Systems. Part II: Multibody Dynamics,” Int. J. Numer. Methods Eng., 67(4), pp. 499-552.

[20] Leyendecker, S., Betsch, P., and Steinmann, P., 2008, "The Discrete Null Space Method for the Energy Consistent Integration of Constrained Mechanical Systems. Part III: Flexible Multibody Dynamics," Multibody Syst. Dyn., 19, pp. $45-72$.

[21] Marsden, J., and Ratiu, T., 1994, Introduction to Mechanics and Symmetry. A Basic Exposition of Classical Mechanical Systems, Texts in Applied Mathematics, Springer, New York, Vol. 17.

[22] Bullo, F., and Lewis, A., 2004, Geometric Control of Mechanical Systems, Springer, New York.

[23] Kirkpatrick, S., Gelatt, C., Jr., and Vecchi, M., 1983, "Optimization by Simulated Annealing," Science, 220(4598), pp. 671-680. 